\title{
A framework to identify modifier genes in patients with Phelan-McDermid syndrome
}

Short running title: Mapping modifier genes in Phelan-McDermid Syndrome

Anne-Claude Tabet $^{1,2,3,4 \pi}$, Thomas Rolland ${ }^{2,3,4 \pi}$, Marie Ducloy ${ }^{2,3,4}$, Jonathan Lévy ${ }^{1}$, Julien Buratti ${ }^{2,3,4}$, Alexandre Mathieu ${ }^{2,3,4}$, Damien Haye ${ }^{1}$, Laurence Perrin ${ }^{1}$, Céline Dupont $^{1}$, Sandrine Passemard ${ }^{1}$, Yline Capri $^{1}$, Alain Verloes ${ }^{1}$, Séverine Drunat ${ }^{1}$, Boris Keren $^{5}$, Cyril Mignot ${ }^{6}$, Isabelle Marey ${ }^{7}$, Aurélia Jacquette ${ }^{7}$, Sandra Whalen ${ }^{7}$, Eva Pipiras $^{8}$, Brigitte Benzacken ${ }^{8}$, Sandra Chantot-Bastaraud $^{9}$, Alexandra Afenjar ${ }^{10}$, Delphine Héron ${ }^{10}$, Cédric Le Caignec ${ }^{11}$, Claire Beneteau ${ }^{11}$, Olivier Pichon ${ }^{11}$, Bertrand Isidor $^{11}$, Albert David ${ }^{11}$, Jean-Michel Dupont ${ }^{12}$, Stephan Kemeny $^{13}$, Laetitia Gouas ${ }^{13}$, Philippe Vago $^{13}$, Anne-Laure Mosca-Boidron ${ }^{14}$, Laurence Faivre ${ }^{15}$, Chantal Missirian $^{16}$, Nicole Philip ${ }^{16}$, Damien Sanlaville ${ }^{17}$, Patrick Edery $^{18}$, Véronique Satre ${ }^{19}$, Charles Coutton $^{19}$, Françoise Devillard ${ }^{19}$, Klaus Dieterich ${ }^{20}$, Marie-Laure Vuillaume ${ }^{21}$, Caroline Rooryck ${ }^{21}$, Didier Lacombe ${ }^{21}$, Lucile Pinson ${ }^{22}$, Vincent Gatinois ${ }^{22}$, Jacques Puechberty $^{22}$, Jean Chiesa ${ }^{23}$, James Lespinasse ${ }^{24}$, Christèle Dubourg ${ }^{25}$, Chloé Quelin ${ }^{25}$, Mélanie Fradin ${ }^{25}$, Hubert Journel ${ }^{26}$, Annick Toutain ${ }^{27}$, Dominique Martin ${ }^{28}$, Abdelamdjid Benmansour ${ }^{1}$, Roberto Toro ${ }^{2,3,4}$, Frédérique Amsellem ${ }^{29}$, Richard Delorme $^{2,3,4,29}$, Thomas Bourgeron ${ }^{2,3,4^{*}}$

${ }^{1}$ Genetics Department, Robert Debré Hospital, APHP, Paris, France,

${ }^{2}$ Human Genetics and Cognitive Functions, Institut Pasteur, Paris, France,

${ }^{3}$ CNRS UMR 3571 Genes, Synapses and Cognition, Institut Pasteur, Paris, France

${ }^{4}$ Université Paris Diderot, Sorbonne Paris Cité, Human Genetics and Cognitive Functions, Paris, France

${ }^{5}$ Cytogenetics Unit, Pitié Salpetrière Hospital, APHP, Paris, France, 
${ }^{6}$ Neurogenetics Unit, Pitié Salpetrière Hospital, APHP, Paris, France,

${ }^{7}$ Clinical Genetics Unit, Pitié Salpetrière Hospital, APHP, Paris, France,

${ }^{8}$ Cytogenetics Unit, Jean Verdier Hospital, APHP, Bondy, France,

${ }^{9}$ Cytogenetics Unit, Trousseau Hospital, APHP, Paris, France,

${ }^{10}$ Clinical Genetics Unit, Trousseau Hospital, APHP, Paris, France,

${ }^{11}$ Clinical Genetics Unit, Nantes Hospital, Nantes, France,

${ }^{12}$ Cytogenetics Unit, Cochin Hospital, APHP, Paris, France,

${ }^{13}$ Genetics Unit, CHU Estaing, Clermont-Ferrand, France,

${ }^{14}$ Cytogenetics Unit, Dijon Hospital, Dijon, France,

${ }^{15}$ Clinical Genetics Unit, Dijon Hospital, Dijon, France,

${ }^{16}$ Genetics Unit, La Timone Hospital, Marseille, France,

${ }^{17}$ Cytogenetics Unit, Lyon Civil Hospital, Lyon, France,

${ }^{18}$ Clinical Genetics Unit, Lyon Civil Hospital, Lyon, France,

${ }^{19}$ Cytogenetics Unit, Grenoble Hospital, Grenoble, France,

${ }^{20}$ Clinical Genetics Unit, Grenoble Hospital, Grenoble, France,

${ }^{21}$ Genetics Unit, Bordeaux Hospital, Bordeaux, France,

${ }^{22}$ Genetics Unit, Montpellier Hospital, Montpellier, France,

${ }^{23}$ Genetics Unit, CHRU Nimes, Nimes, France

${ }^{24}$ Cytogenetics Unit, Chambéry-Hôtel-Dieu Hospital, Chambery, France

${ }^{25}$ Genetics Unit, CHU Rennes, Rennes, France

${ }^{26}$ Genetics Unit, Chubert Hospital, Vannes, France

${ }^{27}$ Genetics Unit, Bretonneau Hospital, Tours, France

${ }^{28}$ Genetics Unit, CH Le Mans, Le Mans, France

${ }^{29}$ Department of Child and Adolescent Psychiatry, Robert Debré Hospital, APHP, Paris,

France

* Corresponding author 
Human Genetics and Cognitive Functions unit, Institut Pasteur, 25 rue du docteur Roux

75015, Paris, France

Phone: 33140613216

Email: thomas.bourgeron@pasteur.fr

I These authors contributed equally to this work 


\section{ABSTRACT}

Phelan-McDermid syndrome (PMS) is characterized by a variety of clinical symptoms with heterogeneous degrees of severity, including intellectual disability, speech impairment, and autism spectrum disorders (ASD). It results from a deletion of the $22 q 13$ locus that in most cases includes the SHANK3 gene. SHANK3 is considered a major gene for PMS, but the factors modulating the severity of the syndrome remain largely unknown. In this study, we investigated 85 PMS patients with different 22q13 rearrangements (78 deletions, 7 duplications). We first explored their clinical features and provide evidence for frequent corpus callosum abnormalities. We then mapped candidate genomic regions at the $22 q 13$ locus associated with risk of clinical features, and suggest a second locus associated with absence of speech. Finally, in some cases, we identified additional rearrangements at loci associated with ASD, potentially modulating the severity of the syndrome. We also report the first SHANK3 deletion transmitted to five affected daughters by a mother without intellectual disability nor ASD, suggesting that some individuals could compensate for such mutations. In summary, we shed light on the genotype-phenotype relationship of PMS, a step towards the identification of compensatory mechanisms for a better prognosis and possibly treatments of patients with neurodevelopmental disorders.

\section{KEYWORDS}

Autism; Phelan-McDermid syndrome; neurodevelopmental disorder; modifier genes; speech 


\section{INTRODUCTION}

Phelan-McDermid syndrome (PMS) is a severe medical condition characterized by hypotonia, global developmental delay, intellectual disability (ID), absent or delayed speech, minor dysmorphic features, and autism spectrum disorders (ASD). ${ }^{1}$ It results from a deletion of the $22 q 13$ locus in the distal part of the long arm of chromosome 22. PMS occurs in $0.2-0.4 \%$ of individuals with neurodevelopmental disorders (NDD), ${ }^{2}$ but the prevalence remains difficult to ascertain due to important biases in the identification of the patients.

In most reported cases, the 22q13 deletion appeared de novo. ${ }^{3}$ The size of the deleted genomic segment varies from hundreds of kilobases $(\mathrm{kb})$ to more than nine megabases $(\mathrm{Mb})$. The mechanisms resulting in the deletion are also highly variable, including simple deletions, unbalanced translocations, ring chromosomes or more complex chromosomal rearrangements. ${ }^{2}$ In the vast majority of the cases, the deletion includes SHANK3, a gene associated with several neuropsychiatric disorders including ID, ASD and schizophrenia. ${ }^{4-6}$ Rare interstitial deletions at $22 q 13$ not encompassing SHANK3 were also observed in patients with features of PMS, suggesting the implication of additional genes in that region or a positional effect influencing SHANK3 expression. ${ }^{7,8}$

SHANK3 codes for a scaffolding protein at the post-synaptic density of glutamatergic synapses, and is known to play a critical role in synaptic function by modulating the formation of dendrites. ${ }^{9}$ Mice lacking Shank3 present alterations in the morphogenesis of the dendritic spines of hippocampal neurons and abnormal synaptic protein levels at the post-synaptic density of glutamatergic synapses. ${ }^{10}$ Studies using human neurons derived from induced pluripotent stem cells (iPSC) of patients with PMS revealed that abnormal networks are reversible by an 
overexpression of the SHANK3 protein or by treatment with insulin growth factor 1 (IGF1)..$^{11}$ SHANK3 mutations can also cause a channelopathy due to a reduction of the hyperpolarization-activated cyclic nucleotide-gated channel proteins $(\mathrm{HCN}$ proteins). ${ }^{12}$ Finally, using iPSCs from patients with ASD and carrying de novo SHANK3 loss-of-function mutations, Darville et al. identified lithium as a factor increasing SHANK3 transcripts. ${ }^{13}$

Patients diagnosed with PMS display a large range of clinical features with various degrees of functional impact. Most patients are dependent on their caregivers. Associated medical or psychiatric comorbidities include seizures (grand mal seizures, focal seizures and absence seizures), renal abnormalities (mainly absent kidney, structural abnormalities of the kidney, hydronephrosis and kidney reflux), cardiac defects (tricuspid valve regurgitation, atrial septal defect, patent ductus arteriosus, and total anomalous pulmonary return), gastrointestinal disorders (intestinal/anal atresia, chronic constipation, gastroesophageal reflux), and ophthalmic features (most often strabismus). ${ }^{14}$ The molecular basis for this clinical heterogeneity is still largely unresolved. To date, at least 13 case series have been published gathering 584 affected patients with PMS. ${ }^{14}$ Only five studies (including 310 subjects) investigated the correlation of clinical features variability and the size of the $22 q 13$ deletion, ${ }^{15-19}$ but the causality remains unclear. In addition, none of these studies explored the role of additional genetic variants ("multiple-hits") in the diversity and severity of the symptoms reported in patients. ${ }^{20,21}$

In the present study, we collected clinical and genomic data from 85 patients carrying an unbalanced genomic rearrangement involving SHANK3 (78 deletions and 7 duplications). We first describe the impact of the size of the $22 q 13$ deletion on the clinical features. Then, we report the first identification of multiple-hits in patients with 
PMS, some affecting known ASD-risk loci that could influence the diversity and severity of the phenotype. Finally, we illustrate the clinical and genomic heterogeneity of individuals carrying $22 q 13$ rearrangements in a family with five affected siblings who inherited a SHANK3 deletion from a mother without ID nor ASD, providing a proof of principle that some individuals could compensate for such mutations.

\section{MATERIALS AND METHODS}

\section{Population}

Eighty-five patients (39 males, 44 females and two fetuses) carrying a genomic rearrangement at the $22 q 13$ locus encompassing SHANK3 were recruited through a French national network of cytogeneticists from 15 centers (ACHROPUCE network). For seven centers, the total number of microarray analyses performed for ID, congenital anomalies or ASD was available to establish a broad estimation of the prevalence of SHANK3 deletions (Table S1). Detailed clinical information was collected for all subjects based on medical records, including perinatal events, growth parameters at birth and during early life of developmental, cognitive and functional development, congenital anomalies, dysmorphic features and main somatic comorbidities (Figure 1, Table S2, Supplementary Materials and Methods). Results from electroencephalograms (EEG) and brain magnetic resonance imaging (MRI) were also gathered for 21 and 35 individuals, respectively. We used the Diagnostic and Statistical Manual of Mental Disorder Fifth edition (DSM-5) criteria for ASD diagnostic, and standardized evaluations including the Autism Diagnostic Interview Revised $(A D I-R)^{22}$ and the Autism Diagnostic Observation Schedule (ADOS-2). ${ }^{23}$ The cognitive level was also measured using Raven's Progressive Matrices for nonverbal intelligence quotient (IQ) and the Peabody picture vocabulary test for verbal 
IQ. The local Institutional Review Boards (IRB) at each clinical center approved the study. For each patient, a parent or legal guardian consented to the study on his or her behalf.

\section{Identification of Genomic Rearrangements}

Genomic data included information on the type of genomic rearrangement at the $22 q 13$ locus, the methods of detection and confirmation, the molecular coordinates and the inheritance status (Table S3). We considered the size and breakpoints of the $22 q 13$ rearrangement only for 74 patients studied using microarray technologies (Figure S1, Table S3).

Quality controls were performed locally in each genetic laboratory (Table S3). Genomic coordinates of each CNV are given according to the hg19 version of the human reference genome. All $22 q 13$ rearrangements were validated in the corresponding clinical center using independent molecular technologies such as $\mathrm{FISH}, \mathrm{qPCR}$ or MLPA. Inheritance of the $22 \mathrm{q} 13$ rearrangement was also determined in the corresponding laboratories.

We mapped all CNVs in the genomes of the patients, and focused on a list of 1,184 candidate genes for neuropsychiatry (NP-genes), which included Class I-III genes, ${ }^{24}$ TADA-65 genes, ${ }^{25}$ the genes from the SFARI database (release from the $9^{\text {th }}$ November 2015; https://gene.sfari.org), and the developmental brain disorder genes (Supplementary Tables 4 and 5). ${ }^{26}$ Four patients (P20, P22, P79 and P81) were not included in the analysis since their arrays did not pass the quality control for a wholegenome analysis. In 14 cases with unbalanced reciprocal translocations (Table S3), we considered the other chromosomal unbalanced segments as additional CNVs. 
The burden of CNVs in patients with PMS was compared to independent cohorts of patients with NDD (Figure S2).

\section{Computational and Statistical Analyses}

Most analyses were performed on genetic and clinical data from 74 patients with array data (67 with a 22q13 deletion, 7 with a duplication), excluding 11 patients for whom only standard cytogenetic data were available (Table S3).

Ward's hierarchical agglomerative clustering analysis was performed using JMP Pro 10.0.2 (http://www.jmp.com) on 40 individuals for whom data on ASD, speech, seizures and deletion size were available (Table S2).

For the mapping of the $22 q 13$ locus, the prevalence of each feature was measured each $50 \mathrm{~kb}$ in sliding windows of $1.5 \mathrm{Mb}$. For each window, we calculated the percentage of individuals carrying a CNV starting in the corresponding interval and showing ASD, absence of speech, ophthalmic features, seizures, gastroesophageal reflux, heart abnormalities or corpus callosum abnormalities. Then, for each feature, we identified the interval defined by the minimal and maximal positions at which the prevalence was higher than the overall prevalence of the feature in the cohort. Finally, the association of the region with the feature was measured by a Fisher's exact test for the enrichment odds ratio (OR) and p-value of individuals having the feature and carrying a CNV starting in the region compared to the rest of the $22 q 13$ locus. To measure the probability to find such regions given the clinical and genetic sampling of the cohort, we developed a bootstrap-based measure of the statistical power. For each feature, we re-shuffled 1,000 times the feature presence/absence of the individuals, and reran our analysis of prevalence. We counted the number of times the enrichment OR was higher than the one 
observed with the original data, and used it as an empirical p-value (hereafter referred to as sampling $p$-value).

\section{RESULTS}

\section{The 22q13 Rearrangements: Prevalence and Clinical Features}

Based on the results of seven clinical centers gathering 18,115 patients with ID, ASD and/or congenital malformations, we could provide a broad estimation of $0.27 \%$ for the prevalence of $22 q 13$ deletions $(0.21$ to $1.38 \%$ depending on the clinical center) (Table S1). Unlike most NDD for which males are significantly more affected than females, we observed a balanced sex ratio with 37 males and 39 females carrying a $22 q 13$ deletion. Clinical features of patients included in this study were similar to those previously reported in larger datasets of patients (Figure 1, Supplementary Materials and Methods). However, for 35 patients, we analyzed the structural brain MRI data and 23 had macroscopic anomalies (65.7\%), consistent with those already described in PMS (Supplementary Materials and Methods). Remarkably, 10 of these patients $(28 \%)$ presented abnormalities of the corpus callosum ranging from thinness to agenesis (Figure S3) indicating that this abnormality is found in a relatively large subgroup of patients with PMS.

\section{The 22q13 Rearrangements: Inheritance and Sizes}

Our cohort included 78 patients carrying a deletion at 22q13 overlapping SHANK3 and seven patients with a duplication (Table S3). The deletions mostly derived from simple de novo deletions (57\%), including two that were mosaic in the somatic cells of the patients (Figure 1). Other de novo chromosomal rearrangements included terminal deletions caused by a ring chromosome 22 in four cases, and deletions 
derived from unbalanced reciprocal translocations in two cases. Altogether, de novo rearrangements accounted for 51 cases (65.4\%). In 12 cases, the deletions resulted from recombined parental balanced translocations or inversions. The size of the deleted segment was highly variable, ranging from $45.8 \mathrm{~kb}$ to $9.1 \mathrm{Mb}$ (Figure S4).

The duplications occurred de novo in 5 cases. For P10, it was inherited from a healthy mother and for P61, inheritance was unknown. Two duplications were in mosaic (P29 and P50), including one case (P29) where the duplication resulted from an unbalanced translocation. The size of the duplicated segment ranged from $96 \mathrm{~kb}$ to $5.8 \mathrm{Mb}$.

\section{Exploratory Cluster Analysis of PMS Clinical Heterogeneity}

To identify homogeneous groups of patients, we performed a hierarchical clustering analysis on 40 individuals for whom information concerning sex and presence or absence of ASD traits, speech and seizures were available. Five clusters summarized the variability (Figure 2). The main separation was based on the size of the deletion. Clusters $\mathrm{C} 1$ (six patients) and C2 (nine patients) were characterized by large deletions of an average of 5 and $7 \mathrm{Mb}$, respectively. These two clusters showed very similar percentages of patients with absence of speech (>80\% in both clusters), but differed specifically for seizures with $0 \%$ and $67 \%$ of the patients in cluster $\mathrm{C} 1$ and C2, respectively. Patients with smaller deletions were divided in three clusters. Clusters C3 (eight patients) and C4 (ten patients) both had deletions of medium size (1.4-1.6Mb), but differed for sex and ASD (C3: 100\% female and 0\% ASD; C4: 100\% males and 60\% ASD). Finally, cluster C5 included seven patients with the smallest deletions and all with ASD and seizures. Therefore, the size of the $22 q 13$ deletion might at least partly explain the presence and severity of the PMS symptoms, 
prompting a more detailed mapping of the genomic regions associated to specific symptoms in order to identify modifier genes.

\section{Association between Clinical Features and Genomic Regions at 22q13}

To map the genomic regions associated to PMS-related features, we measured the prevalence of ASD traits, absence of speech, ophthalmic features, seizures, gastroesophageal reflux, heart abnormalities or corpus callosum abnormalities in sliding windows of $1.5 \mathrm{Mb}$, each $50 \mathrm{~kb}$ in the $22 \mathrm{q} 13$ locus (Figure 3, Materials and Methods). Using this approach, we mapped candidate $22 q 13$ regions conferring a high risk to display specific clinical features, which were statistically assessed for enrichment (odds ratio and p-value) and for statistical power to identify such a region given the sampling of the cohort (sampling p-value, Materials and Methods). We found regions with significantly higher risk for absence of speech, ophthalmic features and gastroesophageal reflux.

The prevalence for absence of speech was $49 \pm 6 \%$ in the cohort, but increased to $80 \%$ for individuals who carry deletions including the genomic segment between position 42.7-46.25 $\mathrm{Mb}$ on chromosome 22 (enrichment $\mathrm{OR}=6.7, \mathrm{P}=0.006$ and sampling $P=0.039$, Figure 3 ). Thirty-eight protein-coding genes are located within this region, including PACSIN2, MPPED1, SULT4A1, ATXN10 and PARVB that are all expressed in the brain and represent compelling candidates for increasing the risk of absence of speech in individuals with PMS.

The overall prevalence of ophthalmic features was $30 \%$, but increased to $70 \%$ when the deletions include the 42.25-44.6 Mb region (enrichment $O R=10.3, P=0.002$ and sampling $P=0.041$, Figure 3 ). Two members of the Parvin gene family, PARVB 
and PARVG, are located at the boundary of this region and code for actin binding proteins.

The prevalence of gastroesophageal reflux was $30 \%$ in the cohort, increased to $70 \%$ when deletions included the $48.9-49.9 \mathrm{Mb}$ region (enrichment $\mathrm{OR}=12.3$, $\mathrm{P}=0.009$ and sampling $\mathrm{P}=0.041)$. This region contains the $F A M 19 A 5$ gene, encoding a small secreted protein mainly expressed in the brain and potentially acting as a modulator of immune response in nervous cells. ${ }^{27}$

For the remaining clinical features associated with PMS (ASD traits, heart abnormalities, seizures and corpus callosum abnormalities), no genomic regions were significantly associated with increased risk and supported by both enrichment and sampling statistical measures, suggesting that other loci or environmental factors could act as modifiers modulating the presence or severity of these features in PMS.

\section{Additional CNVs in Patients with PMS}

In order to test whether other loci could modulate the severity of the clinical features, we systematically identified all the CNVs carried by the patients and affecting the exons of 1,184 candidate neuropsychiatric risk genes (hereafter referred as NPgenes, Table S4, Materials and Methods). Among the 63 patients carrying 22q13.3 deletions and tested using an array technology, 41 carried at least one CNV including at least one exon of a NP-gene (Figure 4, Figure S5, Table S5). Twenty-five patients had only one such CNV, nine had two, and seven had three or more. This burden of CNVs affecting NP-genes did not differ from the one measured in independent cohorts of patients with NDD and tested on similar array platforms (Figure S2). Interestingly, we found five patients who carried CNVs recurrently associated with ASD (Supplementary Materials and Methods). Patient P31 carried a $854 \mathrm{~kb}$ 
duplication at 16p11.2 inherited from a healthy mother, patient P53 carried a $494 \mathrm{~kb}$ deletion at $16 \mathrm{p} 11.2$ of unknown inheritance, patient P60 carried a $300 \mathrm{~kb}$ duplication at $15 q 11.2$ of unknown inheritance, patient P67 carried a 2.7 Mb duplication at 16p12.3 of unknown inheritance, and patient P77 carried a $475 \mathrm{~kb}$ deletion at $15 q 11.2$ inherited from a healthy father.

Out of the 14 patients carrying $22 q 13$ deletions and having seizures, four were carrying an additional CNV covering a known risk gene for epilepsy: KCNT1 (OMIM: 608167), MYT1L (OMIM: 613084), DEAF1 (OMIM: 602635) and SLC25A22 (OMIM: 609302) (Figure 4, Figure S5). For example, patient P8, who was severely affected and unable to speak, had a ring chromosome 22 resulting in a terminal $22 q 13$ deletion of $7.1 \mathrm{Mb}$ and an additional $87 \mathrm{~kb}$ deletion at $9 \mathrm{q} 34$ including KCNT1 that codes for a sodium-activated potassium channel, largely expressed in the nervous system and implicated in autosomal dominant forms of epilepsy.

\section{A Multiplex Family with an Inherited SHANK3 Deletion}

Among the patients included in this study, we identified one female patient (subject III-D, Figure 5) who carried a maternally inherited SHANK3 deletion of $67 \mathrm{~kb}$, removing exons 1-8 of the isoform $A$. In addition, she also carried a $104 \mathrm{~kb}$ deletion of NRXN1 at 2p16.3 (exons 3-5 of the Alpha 2 isoform and exons 3-4 of the Alpha 1 isoform). This proband was a member of a multiplex family that illustrates the heterogeneous clinical severity and the presence of multiple hits in the genome of the patients.

The proband was a 10-year-old female, fourth child (among six) of consanguineous parents (2nd degree). Birth and neonatal parameters were in the normal range. Despite normal development during infancy, the parents reported 
paucity of social interactions, but without stereotyped body movements. The patient exhibited mildly delayed speech, with first words between three and four years old. At eight years old, she was diagnosed with ID, and assessed for cognitive impairment (full scale $I Q=49$, below the first percentile for her age). She did not meet criteria for ASD or any additional axis I psychiatric comorbidities. The patient presented with minor dysmorphic features including a curved forehead with a high implantation of her hair, a short and broad nose with flat nasal tip, long flat philtrum, a thick upper lip with a retrognathy. Concordant with her cognitive defects, she attended a special needs school and acquired basic writing and reading skills.

The father had no personal medical or psychiatric history and carried none of the two CNVs including SHANK3 and NRXN1. In contrast, the mother (subject II-A, Figure 5) had mild learning difficulties related to a borderline IQ (non-verbal IQ=75), but she self-reported no history of speech delay. She carried the two deletions including SHANK3 and NRXN1. The clinical assessment of the mother revealed that she had no significant autistic symptoms, no axis I psychiatric comorbidities and no significant medical history. She shared similar signs of dysmorphism with her daughter.

We also investigated the sisters of the proband (subject III-A, III-B, III-C, III-E and III-F; Figure 5, Supplementary Materials and Methods). Only one girl (III-C) did not carry the SHANK3 deletion. She had a full scale IQ in the normal range (FS$I Q=92$ ). She did not display any significant autistic symptoms (Social Responsiveness Scale total score $<66$ percentile) or any signs of developmental delay (specifically no significant delay in speech development). The four other girls presented either moderate speech delay with first sentences at 4 years (III-A, III-B), or both speech disorder and mild to moderate ID (III-E, III-F). However, none of them 
displayed significant autistic symptoms, nor axis I psychiatric comorbidities and significant medical history. Finally, we investigated one of the maternal uncles (subject II-C, Figure 5), who also carried the SHANK3 deletion and presented mild ID associated with a severe language impairment. He currently has a job dedicated to persons with mental health problems.

Genetic testing showed that the five affected daughters and the maternal uncle carried the $22 q 13$ deletion removing the first part of isoform A of SHANK3. In addition, subjects III-B, III-C, III-D, III-F and II-C also had the 2p16.3 deletion overlapping NRXN1 (Figure 5). All affected subjects carried the partial SHANK3 deletion suggesting its pathogenicity in the syndrome. The $2 \mathrm{p} 16.3$ deletion coexists in three patients with the $22 q 13$ deletion, two with ID and speech delay (III-D and IIIF) and one with only speech delay (III-B). This CNV could act as a modifier factor contributing at least in part to the clinical variability of the syndrome in this family. The unaffected girl (III-C) carried only the NRXN1 deletion, suggesting that, alone, this CNV should not be considered as a risk factor.

\section{DISCUSSION}

\section{Prevalence of PMS, ASD, Seizures and Brain Structural Abnormalities}

Our broad estimation of the prevalence of patients with PMS in patients with NDD is $0.27 \%(0.21-1.38 \%)$, which is similar to the ones reported previously $(0.2-0.4 \%) .{ }^{2} \mathrm{We}$ also observed a balanced sex ratio as previously reported. ${ }^{1}$ Regarding the clinical features (Table S2, Supplementary Materials and Methods), we confirmed previous reports: in most cases, patients with PMS received a diagnosis of NDD, ID and/or speech impairment. 
In our cohort, $50 \%$ of the patients were reported with autistic traits. In the literature, the frequency of patients with PMS diagnosed with ASD varied significantly from one study to another mostly because of inconsistent methods of evaluation. In a report of eight patients, Philippe et al. (2008) did not diagnose ASD in any of the patient. ${ }^{28}$ In contrast, Phelan et al. (2012) diagnosed ASD in 17 out of 18 patients with PMS. ${ }^{1}$ Soorya et al. (2013) showed that 27 out of 32 patients with PMS (84\%) have a diagnosis of ASD when standardized methods are used. ${ }^{16}$ Based on standardized interview with parents/caretakers, a recent study estimated that approximately $50 \%$ of PMS patients met criteria for ASD (21 patients out of 40$){ }^{29}$ Similarly, the prevalence for seizures seems highly heterogeneous among studies since it ranges from 17 to $70 \%$ in multiple case series. ${ }^{30}$ In Kolevson et al. (2014), $25 \%$ of the patients from 13 independent studies $(121 / 482)$ had seizures, ${ }^{14}$ a frequency very similar to this cohort $(24 \%$; 19/78) (Supplementary Materials and Methods).

In the literature, brain structural abnormalities were reported in six studies, with a mean rate of $25 \%(58 / 233)$ of cases. ${ }^{14}$ Hypoplasia of the cerebellar vermis, ${ }^{31,32}$ thin corpus callosum, ${ }^{16,28,31,33}$ and abnormalities of white matter were previously reported. In our cohort, brain MRI data was available for 35 patients with $22 q 13$ deletions and 23 had structural anomalies (65.7\%), consistent with those already described in PMS (Supplementary Materials and Methods).

\section{Impact of the 22q13.3 Deletion Size on the Absence of Speech}

We observed that ASD was associated with smaller deleted segments, and could confirm that absence of speech was associated with large deletions. ${ }^{15,18}$ Similarly, Sarasua et al. (2011) reported that patients with an absence of speech had deletion 
breakpoints at $43.9 \mathrm{Mb}(22 q 13.2)$ or more proximal. $^{15}$ In our study, patients with breakpoint proximal to $46 \mathrm{Mb}$ were more at risk for absence of speech than patients with smaller distal deletions (Figures 2 and 3). Within the 42.7-46.25 Mb interval, several genes are expressed in the brain and are compelling candidate for increasing the risk of absence of speech. PACSIN2 (protein kinase $\mathrm{C}$ and casein kinase substrate in neurons 2) is a member of the pacsin-syndapin-FAP52 gene family and is upregulated upon neuronal differentiation. It has an essential role in the organization of clathrin-mediated membrane endocytosis in neurons. ${ }^{34}$ The function of MPPED1 remains uncharacterized, but it codes for a metallophosphoesterase domain-containing protein highly expressed in the human fetal brain. SULT4A1 codes for a protein of the sulfotransferase family involved in the metabolism of endogenous factors such as hormones, steroids, and monoamine neurotransmitters, as well as drugs and xenobiotics. SULT4A1 is exclusively expressed in neural tissues, is highly conserved, and has been identified in all vertebrate studied so far. Interestingly, zebrafish carrying homozygous SULT4A1 mutations exhibit excessively sedentary behavior during the day, ${ }^{35}$ suggesting a role of this gene in regulating behavior. The function of $A T X N 10$ remains largely unknown, but expanded ATTCT pentanucleotide repeats in intron 9 of the gene cause a rare form of spinocerebellar ataxia (SCA10) characterized by cerebellar ataxia and epilepsy. ${ }^{36}$

Interestingly, the region associated with absence of speech did not include WNT7B, a member of the WNT signaling molecule involved in the formation of the central nervous system vascular endothelium ${ }^{37}$ as well as in dendrite development. ${ }^{38}$ In contrast, this region includes the minimal interval of interstitial $22 q 13$ deletions (not involving SHANK3) causing clinical features common to $\mathrm{PMS}^{7}$ and including SULT4A1 and PARVB. PARVB is not highly expressed in the brain, but it codes for 
an actin-binding protein that interacts with ARHGEF6, a protein coded by an X-linked gene mutated in patients with ID. ${ }^{39}$

\section{Multiple Hits in Patients with PMS}

To our knowledge, this is the first report investigating the presence of multiple-hits in patients with PMS. Among the 63 patients with array results, 41 carried at least one additional CNV encompassing exonic sequences of one NP-gene. It is important to consider that a duplication of a gene might not be causative and therefore CNVs affecting NP-genes might not always be deleterious. Nevertheless, we found 16p11.2 CNVs (one deletion and one duplication) in two independent patients (P32 and P59), which are known to increase risk of NDD and are rare in the general population $(0.03 \%) .^{2}$ The finding of two cases with such $C N V s$ is intriguing and raises question of the genetic architecture of PMS, and by extension on the cumulative genetic risk factors in NDD. ${ }^{40}$ In addition, we observed abnormal gene dosage of risk-genes for epilepsy such as KCNT1, MYT1L, DEAF1 and SLC25A22 in patients whom had lifetime history of seizures. However, at that stage, we cannot conclude on the causative effect of such CNVs since we also observed patients with CNVs affecting risk-genes for epilepsy (such as KANSL1 or NRXN1), and a lifetime absence of epilepsy. In summary, multiple-hits exist in patients with PMS, but their impact on the severity of the symptoms remains to be determined in larger cohorts of wellphenotyped patients with more extensive genetic profiles using whole exome/genome sequencing.

\section{Conclusions}


Our study confirms previous findings regarding the impact of the $22 \mathrm{q} 13$ deletion on several clinical features such as absence of speech and ASD. We also identified one mother without ID nor ASD carrying a SHANK3 deletion, providing the first proof of principle that some individuals could be resilient for such mutations. Larger cohorts of individuals carrying 22q13 deletions (including interstitial deletions not affecting SHANK3) with in-depth phenotyping and whole genome sequencing data should allow us to identify modifier genes. Such genes and pathways would participate to our understanding of the etiology of PMS and could represent new relevant drug targets.

\section{ACKNOWLEDGEMENTS}

We are grateful to the families for participating in this study. We thank Isabelle CloëzTayarani, Claire Leblond, Ksenia Bagrintseva and Gaël Millot for helpful discussions and careful reading of the manuscript.

The authors declare no competing interests.

\section{REFERENCES}

1. Phelan K, McDermid HE. The 22q13.3 Deletion Syndrome (Phelan-McDermid Syndrome). Mol Syndromol. 2012;2(3-5):186-201. doi:000334260.

2. Coe BP, Witherspoon K, Rosenfeld JA, et al. Refining analyses of copy number variation identifies specific genes associated with developmental delay. Nat Genet. 2014;46(10):1063-1071. doi:10.1038/ng.3092. 
3. Bonaglia MC, Giorda R, Beri S, et al. Molecular mechanisms generating and stabilizing terminal 22q13 deletions in 44 subjects with Phelan/McDermid syndrome. PLoS Genet. 2011;7(7):e1002173.

doi:10.1371/journal.pgen.1002173.

4. Guilmatre A, Huguet G, Delorme R, Bourgeron T. The emerging role of SHANK genes in neuropsychiatric disorders. Dev Neurobiol. 2014;74(2):113-122. doi:10.1002/dneu.22128.

5. Leblond CS, Nava C, Polge A, et al. Meta-analysis of SHANK Mutations in Autism Spectrum Disorders: a gradient of severity in cognitive impairments. PLoS Genet. 2014;10(9):e1004580. doi:10.1371/journal.pgen.1004580.

6. Durand CM, Betancur C, Boeckers TM, et al. Mutations in the gene encoding the synaptic scaffolding protein SHANK3 are associated with autism spectrum disorders. Nat Genet. 2007;39(1):25-27. doi:10.1038/ng1933.

7. Disciglio V, Lo Rizzo C, Mencarelli MA, et al. Interstitial 22q13 deletions not involving SHANK3 gene: A new contiguous gene syndrome. Am J Med Genet Part A. 2014;164(7):1666-1676. doi:10.1002/ajmg.a.36513.

8. Wilson HL, Crolla JA, Walker D, et al. Interstitial 22q13 deletions: genes other than SHANK3 have major effects on cognitive and language development. Eur J Hum Genet. 2008;16(11):1301-1310. doi:10.1038/ejhg.2008.107.

9. Monteiro P, Feng G. SHANK proteins: roles at the synapse and in autism spectrum disorder. Nat Rev Neurosci. 2017;18(3):147-157. doi:10.1038/nrn.2016.183.

10. Peça J, Feliciano C, Ting JT, et al. Shank3 mutant mice display autistic-like behaviours and striatal dysfunction. Nature. 2011;472(7344):437-442. doi:10.1038/nature09965. 
11. Shcheglovitov A, Shcheglovitova O, Yazawa M, et al. SHANK3 and IGF1 restore synaptic deficits in neurons from $22 q 13$ deletion syndrome patients. Nature. 2013;503(7475):267-271. doi:10.1038/nature12618.

12. Yi F, Danko T, Botelho SC, et al. Autism-associated SHANK3 haploinsufficiency causes Ih channelopathy in human neurons. Science. 2016;2669:1-22. doi:10.1126/science.aaf2669.

13. Darville H, Poulet A, Rodet-Amsellem F, et al. Human Pluripotent Stem Cellderived Cortical Neurons for High Throughput Medication Screening in Autism: A Proof of Concept Study in SHANK3 Haploinsufficiency Syndrome. EBioMedicine. 2016;9:293-305. doi:10.1016/j.ebiom.2016.05.032.

14. Kolevzon A, Angarita B, Bush L, et al. Phelan-McDermid syndrome: a review of the literature and practice parameters for medical assessment and monitoring. J Neurodev Disord. 2014;6(1):39. doi:10.1186/1866-1955-6-39.

15. Sarasua SM, Dwivedi A, Boccuto L, et al. Association between deletion size and important phenotypes expands the genomic region of interest in PhelanMcDermid syndrome (22q13 deletion syndrome). J Med Genet. 2011;48(11):761-766. doi:10.1136/jmedgenet-2011-100225.

16. Soorya L, Kolevzon A, Zweifach J, et al. Prospective investigation of autism and genotype-phenotype correlations in 22q13 deletion syndrome and SHANK3 deficiency. Mol Autism. 2013;4(1):18. doi:10.1186/2040-2392-4-18.

17. Sarasua SM, Dwivedi A, Boccuto L, et al. 22q13.2q13.32 genomic regions associated with severity of speech delay, developmental delay, and physical features in Phelan-McDermid syndrome. Genet Med. 2014;16(4):318-328. doi:10.1038/gim.2013.144.

18. Sarasua SM, Boccuto L, Sharp JL, et al. Clinical and genomic evaluation of 
201 patients with Phelan-McDermid syndrome. Hum Genet. 2014;133(7):847859. doi:10.1007/s00439-014-1423-7.

19. Dhar SU, Del Gaudio D, German JR, et al. 22q13.3 Deletion syndrome: Clinical and molecular analysis using array CGH. Am J Med Genet Part A. 2010;152(3):573-581. doi:10.1002/ajmg.a.33253.

20. Leblond CS, Heinrich J, Delorme R, et al. Genetic and functional analyses of SHANK2 mutations suggest a multiple hit model of autism spectrum disorders. PLoS Genet. 2012;8(2):e1002521. doi:10.1371/journal.pgen.1002521.

21. Girirajan S, Rosenfeld JA, Cooper GM, et al. A recurrent 16p12.1 microdeletion supports a two-hit model for severe developmental delay. Nat Genet. 2010;42(3):203-209. doi:10.1038/ng.534.

22. Lord C, Rutter M, Le Couteur A. Autism Diagnostic Interview-Revised: A revised version of a diagnostic interview for caregivers of individuals with possible pervasive developmental disorders. J Autism Dev Disord. 1994;24(5):659-685. doi:10.1007/BF02172145.

23. Lord C, Risi S, Lambrecht L, et al. Autism Diagnostic Observation Schedule (ADOS). J Autism Dev Disord. 2000;30(3):205-223. doi:10.1007/BF02211841.

24. Yuen RK, Thiruvahindrapuram B, Merico D, et al. Whole-genome sequencing of quartet families with autism spectrum disorder. Nat Med. 2015;21(2):185191. doi:10.1038/nm.3792.

25. Sanders SJ, He X, Willsey AJ, et al. Insights into Autism Spectrum Disorder Genomic Architecture and Biology from 71 Risk Loci. Neuron. 2015;87(6):1215-1233. doi:10.1016/j.neuron.2015.09.016.

26. Gonzalez-Mantilla AJ, Moreno-De-Luca A, Ledbetter DH, Martin CL. A CrossDisorder Method to Identify Novel Candidate Genes for Developmental Brain 
Disorders. JAMA Psychiatry. 2016;17837(3):1-9.

doi:10.1001/jamapsychiatry.2015.2692.

27. Tom Tang Y, Emtage P, Funk WD, et al. TAFA: A novel secreted family with conserved cysteine residues and restricted expression in the brain. Genomics. 2004;83(4):727-734. doi:10.1016/j.ygeno.2003.10.006.

28. Philippe A, Boddaert N, Vaivre-Douret L, et al. Neurobehavioral profile and brain imaging study of the 22q13.3 deletion syndrome in childhood. Pediatrics. 2008;122(2):e376-82. doi:10.1542/peds.2007-2584.

29. Oberman LM, Boccuto L, Cascio L, Sarasua S, Kaufmann WE. Autism spectrum disorder in Phelan-McDermid syndrome: initial characterization and genotype-phenotype correlations. Orphanet J Rare Dis. 2015;10(1):105. doi:10.1186/s13023-015-0323-9.

30. Holder JL, Quach MM. The spectrum of epilepsy and electroencephalographic abnormalities due to SHANK3 loss-of-function mutations. Epilepsia. 2016;57(10):1651-1659. doi:10.1111/epi.13506.

31. Aldinger KA, Kogan J, Kimonis V, et al. Cerebellar and posterior fossa malformations in patients with autism-associated chromosome $22 q 13$ terminal deletion. Am J Med Genet Part A. 2013;161(1):131-136. doi:10.1002/ajmg.a.35700.

32. Egger JI, Zwanenburg RJ, van Ravenswaaij-Arts CM, Kleefstra T, Verhoeven WM. Neuropsychological phenotype and psychopathology in seven adult patients with Phelan-McDermid syndrome: Implications for treatment strategy. Genes Brain Behav. 2016:n/a-n/a. doi:10.1111/gbb.12285.

33. Lindquist SG, Kirchhoff M, Lundsteen C, et al. Further delineation of the 22q13 deletion syndrome. Clin Dysmorphol. 2005;14(2):55-60. 
doi:10.1097/00019605-200504000-00001.

34. Sumoy L, Pluvinet R, Andreu N, Estivill X, Escarceller M. PACSIN 3 is a novel $\mathrm{SH} 3$ domain cytoplasmic adapter protein of the pacsin-syndapin-FAP52 gene family. Gene. 2001;262(1-2):199-205. doi:10.1016/S0378-1119(00)00531-X.

35. Crittenden F, Thomas HR, Parant JM, Falany CN. Activity suppression behavior phenotype in SULT4A1 frameshift mutant zebrafish. Drug Metab Dispos. 2015;43(7):1037-1044. doi:10.1124/dmd.115.064485.

36. Teive HA, Munhoz RP, Arruda WO, Raskin S, Werneck LC, Ashizawa T. Spinocerebellar ataxia type 10 - A review. Park Relat Disord. 2011;17(9):655661. doi:10.1016/j.parkreldis.2011.04.001.

37. Stenman J, Rajagopal J, Carroll T, Ishibashi M, McMahon J, McMahon A. Canonical Wnt Signaling Regulates Organ-Specific Assembly and Differentation of CNS Vasculature. Science. 2008;322(5905):1247-1250.

38. Rosso SB, Sussman D, Wynshaw-Boris A, Salinas PC. Wnt signaling through Dishevelled, Rac and JNK regulates dendritic development. Nat Neurosci. 2005;8(1):34-42. doi:10.1038/nn1374.

39. Rosenberger G, Jantke I, Gal A, Kutsche K. Interaction of alphaPIX (ARHGEF6) with beta-parvin (PARVB) suggests an involvement of alphaPIX in integrin-mediated signaling. Hum Mol Genet. 2003;12(2):155-167. doi:10.1093/hmg/ddg019.

40. Bourgeron T. From the genetic architecture to synaptic plasticity in autism spectrum disorder. Nat Rev Neurosci. 2015;16(9):551-563.

doi:10.1038/nrn3992. 


\section{FIGURE LEGENDS}

Figure 1. Clinical and genetic description of the cohort. (A) Spider plot of the clinical profile of the patients with PMS as a percentage of cases having each feature over those tested. (B) Mechanisms and inheritance of identified $22 q 13 \mathrm{CNVs}$ in the cohort. ABCR: apparently balanced chromosomal rearrangement; der(22): derived from unbalanced reciprocal translocation; ring(22): caused by a ring chromosome 22.

Figure 2. Multivariate analysis of the $22 q 13$ deletion size, sex and clinical features of patients. (A) Hierarchical clustering based on multivariate analysis shows five clusters. (B) Tree view showing the five clusters of patients and the main genomic and clinical features of each cluster.

Figure 3. Mapping of genomic regions at $22 q 13$ associated with high risk of presenting clinical features. (A) Prevalence is measured each $50 \mathrm{~kb}$, within overlapping windows of $1.5 \mathrm{Mb}$ (Materials and Methods). Dashed lines represent the global prevalence of each feature, measured as the fraction of patients with $22 q 13$ deletions presenting the feature over all patients with $22 q 13$ deletions. Dotted lines and colored areas represent standard errors of the proportion. Black solid lines show the numbers of informative patients for each window, and black dotted lines correspond to the minimum number of individuals required by window $(n=3)$ to reduce interpretation biases. (B) The $22 q 13$ locus is represented with the genes (block: exon, line: intron, arrow: strand) and the regions corresponding to a higher than global prevalence for each feature (top). Regions significantly associated with 
absence of speech and ophthalmic features are also shown in more details (bottom).

Color code similar to $(A)$.

Figure 4. Additional CNVs identified in the cohort and including at least one NP-gene. Deletions (red) and duplications (blue) are represented for each patient and each NP-gene.

Figure 5. Multiplex family with inherited SHANK3 and NRXN1 deletions. (A) The nine studied individuals are displayed in the pedigree of the family. (B) CNVs detected by OmniExpress Illumina arrays and including the NRXN1 and SHANK3 genes (log2 scale). 


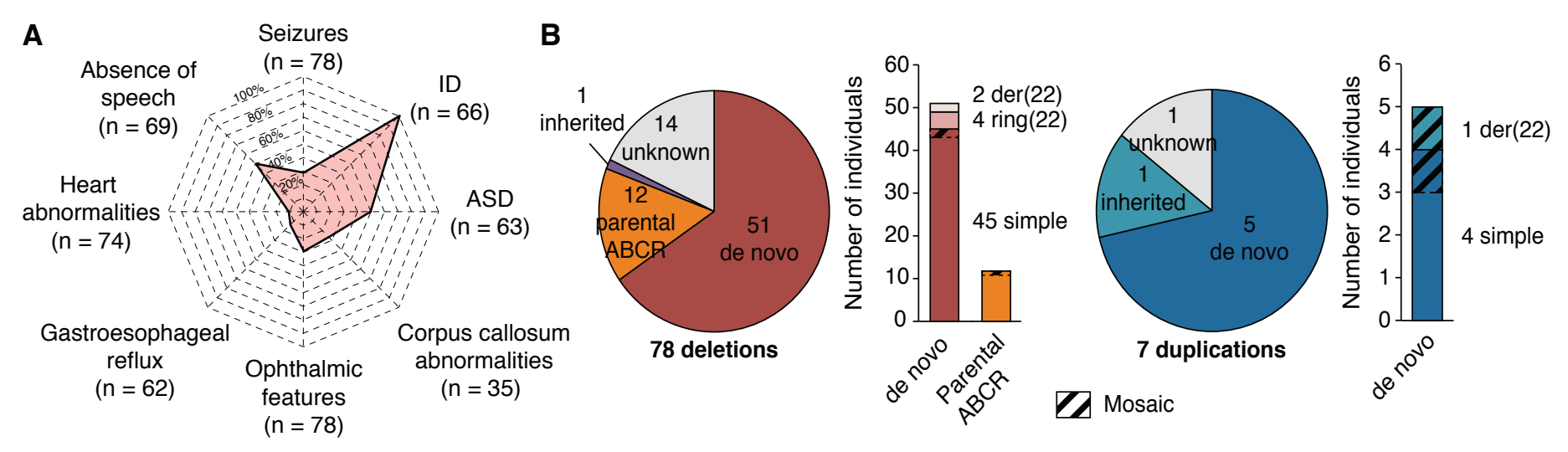




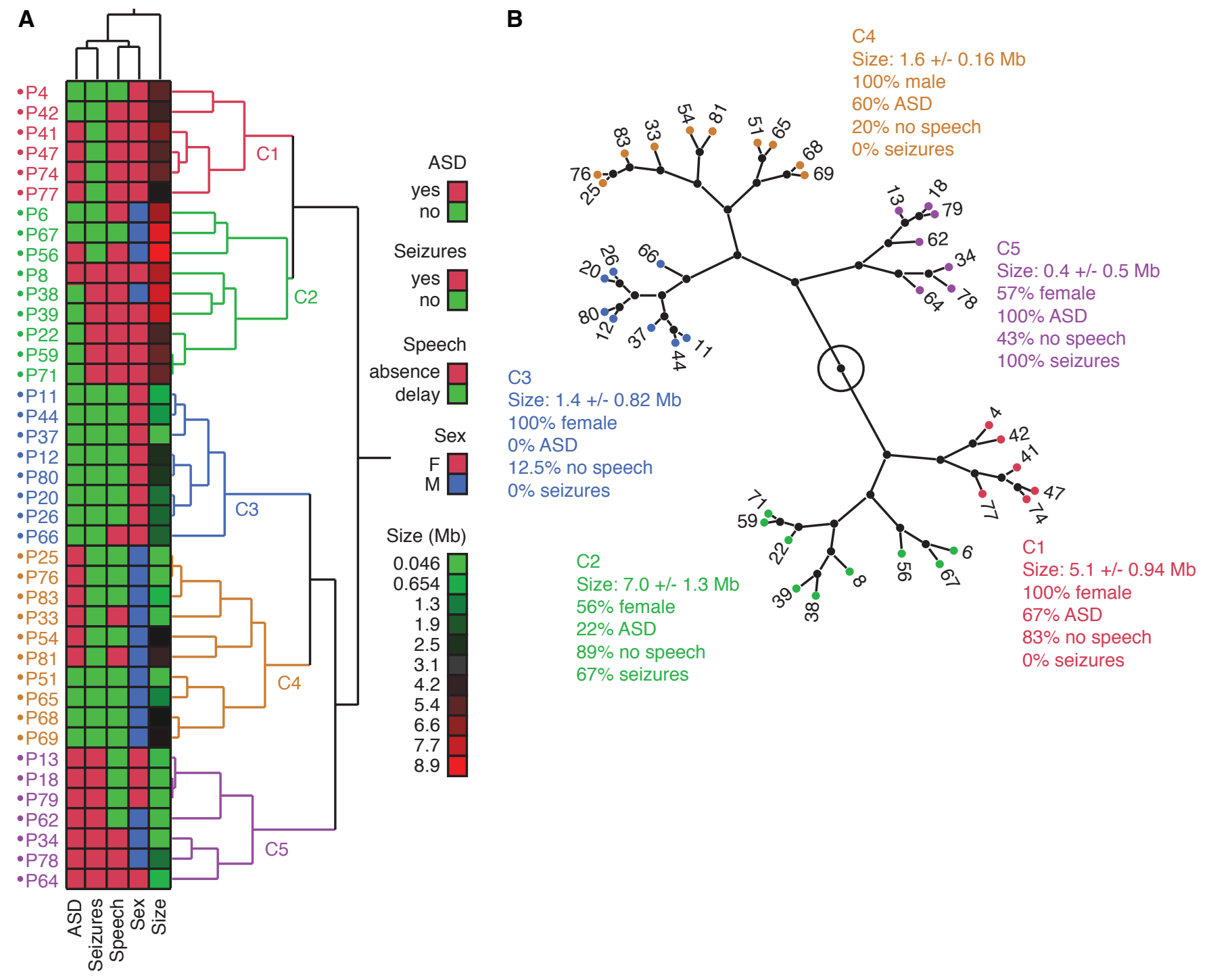




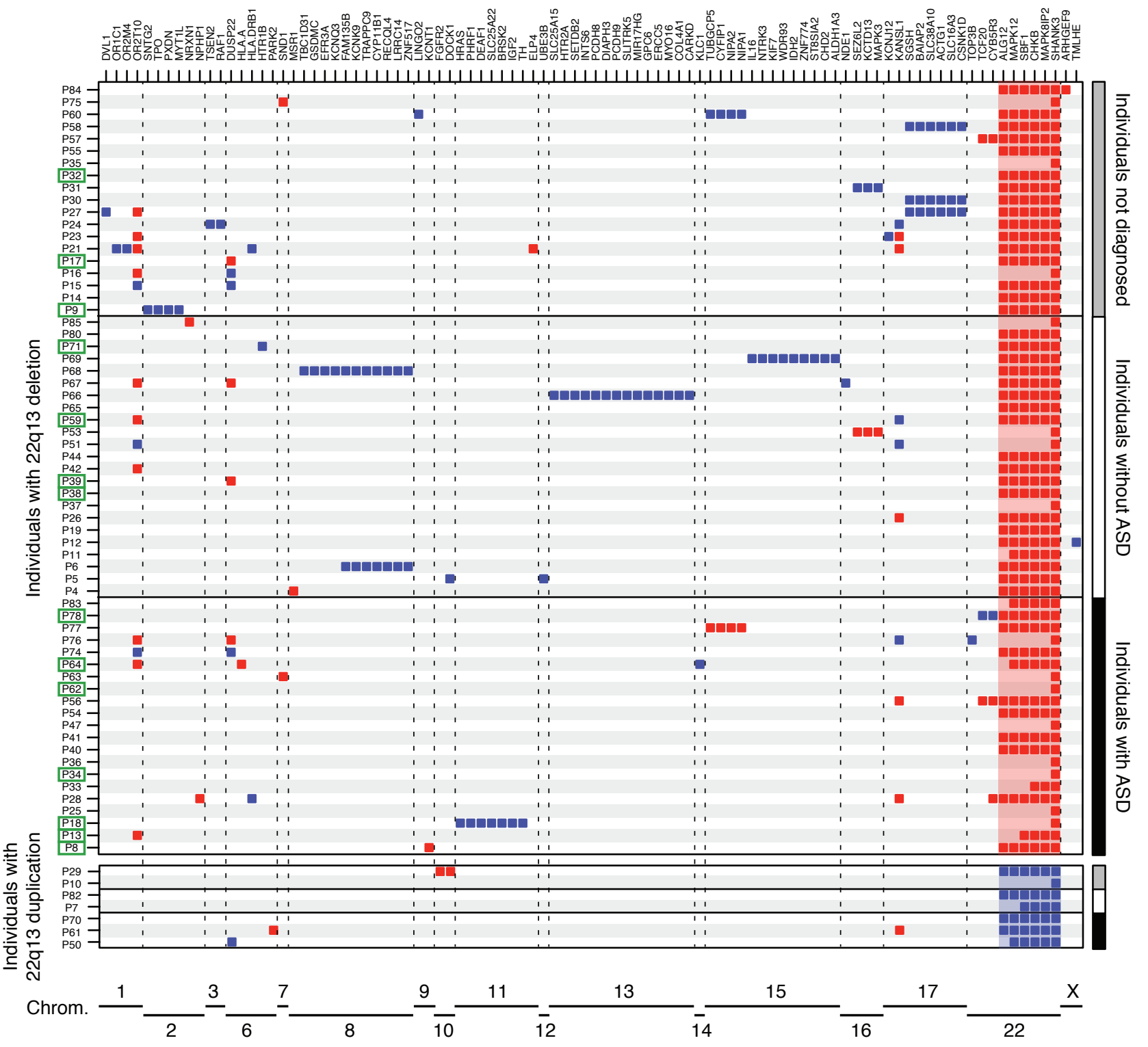




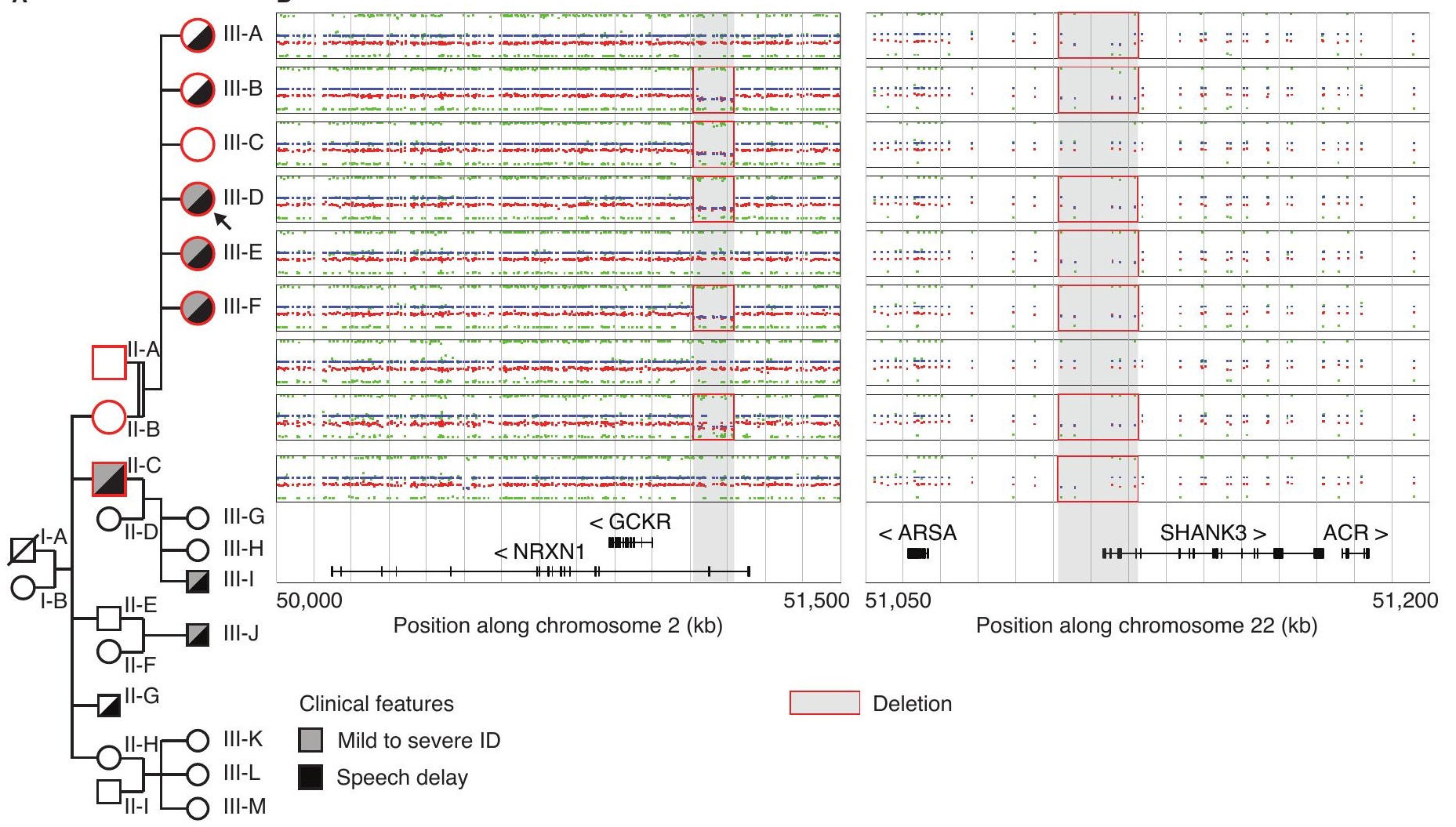

